\section{JTI}

JOURNAL OF

TRAUMA AND INJURY

\title{
Extra-Pericardial Tamponade due to Internal Thoracic Artery Rupture after Blunt Trauma: A Case Report
}

\author{
Dongsub Noh, M.D., Sung Wook Chang, M.D., Ph.D., Dae Sung Ma, M.D. \\ Trauma Center, Department of Thoracic and Cardiovascular Surgery, Dankook University \\ Hospital, Cheonan, Korea
}

Received: June 21, 2021

Revised: August 16, 2021

Accepted: August 20, 2021

\section{Correspondence to}

Dae Sung Ma, M.D.

Trauma Center, Department of Thoracic and Cardiovascular Surgery, Dankook

University Hospital, 201 Manghyang-ro, Dongnam-gu, Cheonan 31116, Korea

Tel: +82-41-550-7640

Fax: +82-41-550-7060

E-mail:pegamu@naver.com

ORCID: https://orcid.org/0000-0001-

7521-3949

Cardiac tamponade is an acute life-threatening condition that predominantly involves the intra-pericardial space; however, an expanding mediastinal hematoma can also sometimes cause cardiac tamponade. Here we describe the case of a 45 -year-old male driver in whom a traffic accident resulted in rupture of the left internal thoracic artery (ITA), extra-pericardial hematoma, and sternal fracture. After resuscitation, he was scheduled to undergo angio-embolization to repair the ruptured left ITA, but he suddenly developed cardiac tamponade that required a decompressive sternotomy. Nevertheless, the patient had an uncomplicated recovery, and this case suggests that extra-pericardial cardiac tamponade should be considered as a possible consequence of retro-sternal hematoma due to traumatic ITA rupture.

Keywords: Blunt injury; Internal thoracic arteries; Cardiac tamponade

\section{INTRODUCTION}

Traumatic internal thoracic artery (ITA) injury is exceedingly rare; it occurs due to blunt trauma in most cases and is accompanied by injuries such as rib fracture (17\%) and sternal fracture (17\%) [1]. Traumatic ITA injury typically presents as hemothorax, anterior mediastinal hematoma, or pseudoaneurysm, and very rarely presents as extra-pleural hematoma, extra-pericardial hematoma, or arteriovenous fistula. Notably, most of these conditions are managed by angio-embolization [1]. Nevertheless, a growing extra-pericardial hematoma can result in cardiac arrest and requires surgical treatment [2]. Here, we describe the successful management of extra-pericardial cardiac tamponade caused by a large retro-sternal hematoma due to traumatic rupture of the ITA.

This is an Open Access article distributed under the terms of the Creative Commons Attribution Non-Commercial License (http://creativecommons.org/licenses/by-nc/4.0/) which permits unrestricted noncommercial use, distribution, and reproduction in any medium, provided the original work is properly cited. 


\section{JTI}

\section{CASE REPORT}

A 45-year-old male driver was transferred to Regional Trauma Center of Dankook University Hospital after he had crashed into a fence. At presentation, he was mildly drowsy and had sustained an approximately $20 \mathrm{~cm}$ long deep laceration on his right knee, and his distal femur was exposed. His vital signs were a blood pressure of 77/56 $\mathrm{mmHg}$, a pulse rate of 103 beats/minute, a respiratory rate of 22 breaths/minute, and a digital oxygen saturation of $98 \%$. The right dorsalis pedis pulse was weak and his Glasgow Coma Scale score was 14 (E3V5M6). An extended focused assessment with sonography did not show the sliding sign, which is a characteristic of left
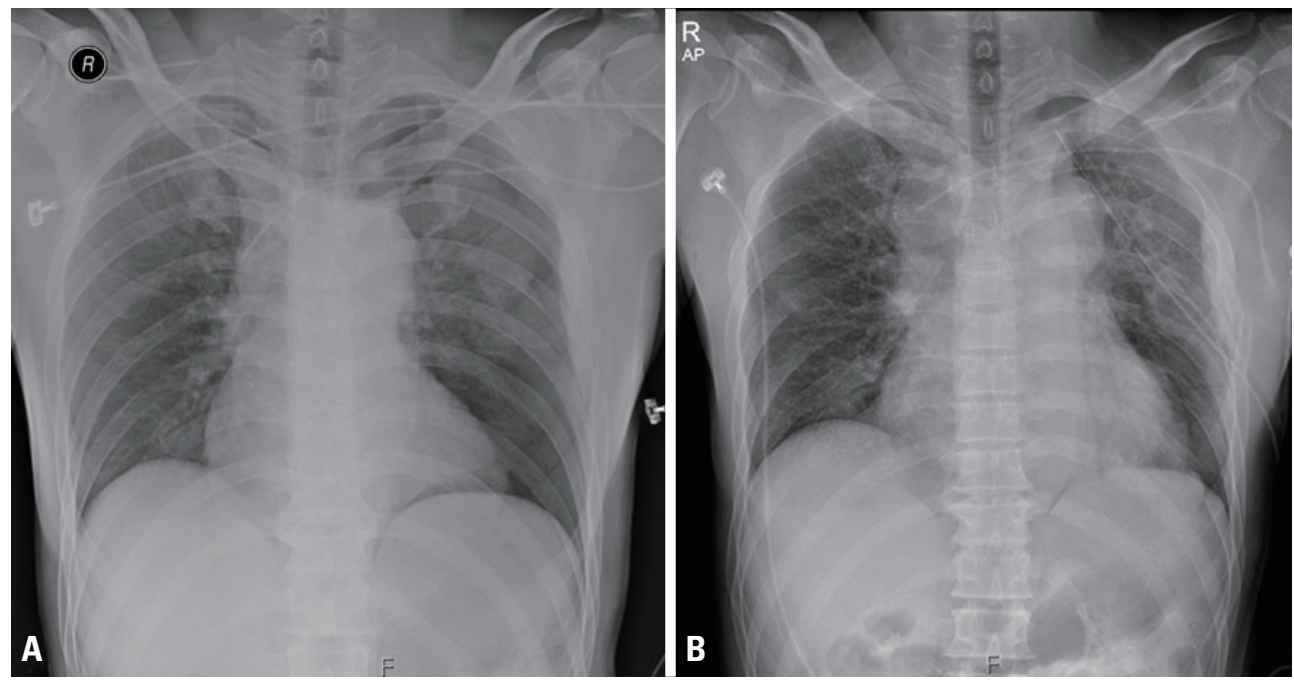

Fig. 1. (A) Left pneumothorax, as seen on the initial chest X-ray. (B) Mediastinal shadow widening was observed on computed tomography.

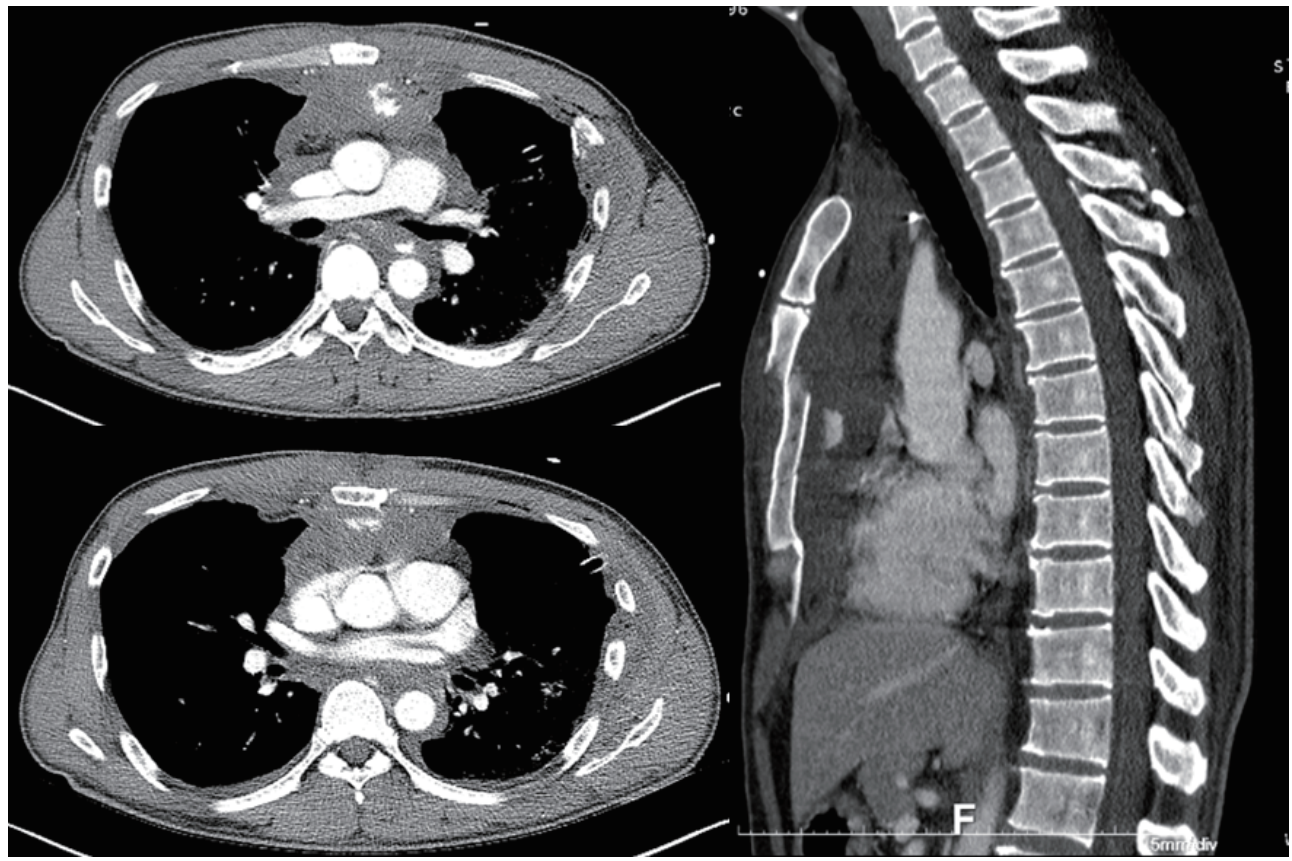

Fig. 2. Chest computed tomography showed active bleeding from the left internal thoracic artery in the anterior mediastinum, along with a sternal fracture. 
hemithorax, and was otherwise unremarkable; however, a chest X-ray examination revealed left pneumothorax (Fig. 1A). After resuscitation with two units of red blood cells and a left-sided closed thoracostomy, his blood pressure increased to $90 / 42 \mathrm{mmHg}$. Although computed tomography (CT) of the brain and the abdomen showed no specific injuries related to this trauma, contrast-enhanced chest CT showed active bleeding from the left ITA in the anterior mediastinum along with a multiple-segment sternal fracture (Fig. 2). Therefore, angiographic embolization of the left ITA and evaluation of the injured artery around the right knee were planned. However, while waiting for angio-embolization, his vital signs deteriorated to a blood pressure of $67 / 32 \mathrm{mmHg}$ and a pulse rate of 120 beats/ minute, and a follow-up chest X-ray showed widening of the mediastinal shadow (Fig. 1B). Fluid management and blood transfusion did not restore his blood pressure and pulse rate. Based on his deterioration, we reasoned that the active bleeding from the ruptured left ITA could have led to extra-pericardial cardiac tamponade. Therefore, rather than angio-embolization of the ITA, we performed a median sternotomy, both to immediately relieve the tamponade by removing the substernal hematoma and to ligate the ruptured ITA. When sternotomy was performed, we found two sites of transverse fractures on the sternum with bone bleeding, a large hematoma compressing the heart, and active bleeding from two rupture sites of the ITA. Removal of the hematoma (about 2,000 mL) restored the patient's vital signs and the proximal part of the left ITA was ligated. The pericardium was opened, and a gross examination did not reveal any injuries to the heart or the great vessels. After controlling bleeding, the sternotomy site was fixed with SternaLock ${ }^{\circledR}$ (Biomet Microfixation Inc., Jacksonville, FL, USA), and the sternotomy area was fixed with a figure-of-eight suture using steel wires (Fig. 3). The patient's immediate postoperative course was uneventful, and he underwent knee repair surgery 3 days later. He was discharged after a 3-month hospital stay.

\section{DISCUSSION}

Cardiac tamponade is an acute, life-threatening condition

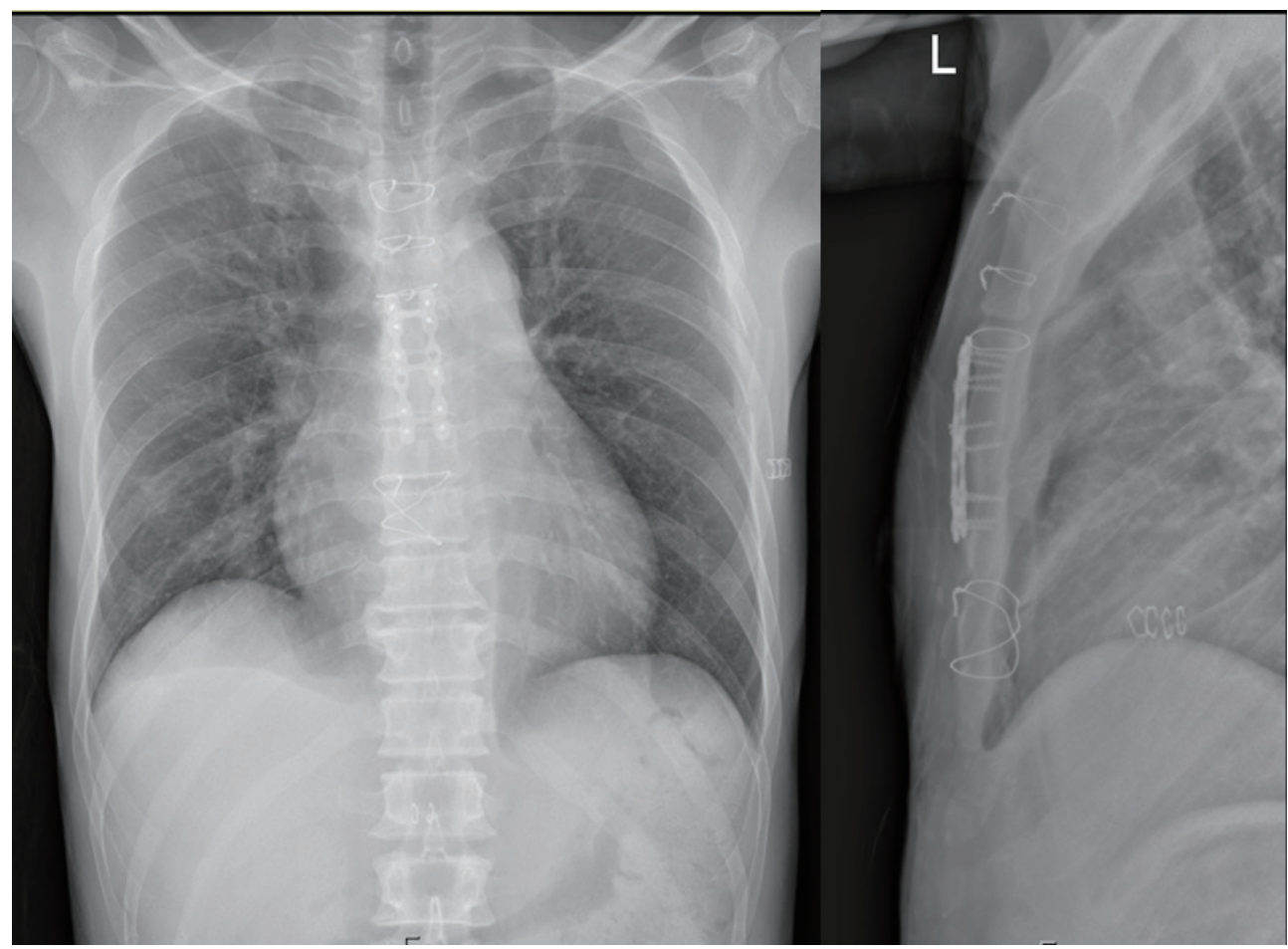

Fig. 3. The fractured sternum was fixed using SternaLock ${ }^{\circledR}$ (Biomet Microfixation Inc., Jacksonville, FL, USA), a steel wire, and figure-of-eight sutures. 
wherein fluid or blood accumulation in the pericardial space leads to an elevation of pericardial pressure and abnormal relaxation of the heart. Although cardiac tamponade typically involves the intra-pericardial space, a mediastinal hematoma can also lead to cardiac tamponade [2]. Cardiac tamponade after blunt chest trauma is rare, but approximately $80 \%$ of cases of traumatic cardiac tamponade are missed during the initial assessment [3]. As traumatic pericardial or extra-pericardial tamponade can be lethal, physicians must be aware of the possibility of tamponade in cases of trauma to the anterior chest wall $[2,4,5]$.

The internal thoracic vessels, including the ITA, lie lateral to the sternum and run vertically downward and posterior to the costal cartilage, resting upon the pleura and the pericardium. Thus, traumatic ITA rupture can cause extra-pericardial cardiac tamponade [5], and active contrast leakage at the rupture can be easily detected on CT $[1,2]$. Although angiographic embolization should always be considered before surgery in physiologically stable patients [1], simple decompressive sternotomy is the treatment of choice for unstable extra-pericardial cardiac tamponade, as it can immediately relieve the pressure on the heart [4]. In our case, CT showed a substernal hematoma and bleeding from the ITA, and cardiac tamponade due to an expanding extra-pericardial hematoma was deemed to be the cause of the patient's deteriorating condition and lack of response to fluid management and blood transfusion. Importantly, prompt surgical management of the hematoma resulted in stabilization of the patient's vital signs.

Angio-embolization is an effective, efficient, and safe treatment modality, but $45 \%$ of all patients also require surgical management [1] because, given a flow rate of $150 \mathrm{~mL} /$ minute, traumatic ITA rupture could lead to life-threatening hemorrhage within a few minutes $[1,4,6]$. Furthermore, a large mediastinal hematoma could cause hemodynamic instability due to both blood loss and the compression of vascular structures, such as the heart [7]. In this case, the position of the retro-sternal hematoma and high blood flow through the ITA resulted in extra-pericardial tamponade.

To summarize, we describe a rare case of extra-pericardial tamponade after blunt chest trauma, wherein the latter caused a multiple-segment sternal fracture and a retro-sternal hematoma due to rupture of the left ITA. Thus, extra-pericardial cardiac tamponade should be considered as a possible consequence of retro-sternal hematoma due to traumatic ITA rupture.

\section{REFERENCES}

1. Chen JM, Lv J, Ma K, Yan J. Assessment of internal mammary artery injury after blunt chest trauma: a literature review. J Zhejiang Univ Sci B 2014;15:864-9.

2. Arabi RI, Aljudaibi A, Althumali AA, Rajb BS, Arja RD. Traumatic retrosternal hematoma leading to extra-pericardial cardiac tamponade-case report. Int J Surg Case Rep 2019;61:30-2.

3. Bleetman A, Kasem H, Crawford R. Review of emergency thoracotomy for chest injuries in patients attending a UK Accident and Emergency Department. Injury 1996;27:129-32.

4. Parri SNF, Guiducci GM, Kawamukai K, Tugnoli G. Unexpected cardiac arrest due to extrapericardial tamponade: beware of hemomediastinum! Emerg Med 2015;5:5.

5. Hajjar RJ, Rose GA, Madsen JC, Levine RA, DeSanctis RW. Extrapericardial cardiac tamponade after blunt chest trauma. Am Heart J 1995;130:620-1.

6. Ritter DC, Chang FC. Delayed hemothorax resulting from stab wounds to the internal mammary artery. J Trauma 1995;39:586-9.

7. Cardinale L, Cataldi A, Giardino R, Prato A, Volpicelli G. Delayed presentation of a huge mediastinal hematoma after blunt chest trauma with extrapericardial cardiac tamponade: a multiphase-multidetector spiral computed tomography study. J Trauma 2010;69:996. 